\title{
An uniform model for Dark Matter and Dark Energy
}

\author{
George N. Izmaïlov
}

Moscow Aviation Institute (National Research University), Moscow, Russia izmailov@mai.ru

\begin{abstract}
In this paper, after reviewing some of the most important concepts about Dark Matter and methods of its registration, in particular by using SQUIDs, a toy uniform model for Dark Matter and Dark Energy is proposed. In the frame of the model Dark Matter particles is interpreted as excitations of Dark Energy field. The devices based on SQUID, in particular the SQUIDparamagnetic absorber and the SQUID-magnetostrictor systems, both suitable for investigations of above problems, are considered. Estimates, is carried out within this model, indicate the possibility of experimental detection of the "ether wind" pressure, created by the non-corpuscular incoming flow, corresponding to the galactic orbital motion of the Earth.
\end{abstract}

Keywords: Dark Matter, Dark Energy, magnetic moment, SQUID, magnetostrictor.

\section{Introduction}

The nature of dark matter (DM) is one of the most urgent unsolved problems in modern physics, so physicists persistently try to find signs of elusive dark matter that tugs on everything but emits no light. History of observations and theoretical arguments for the DM existence are described in a recent review [1]. Besides large number of independent astrophysical and cosmological observations, such as galaxy rotation curves [2], large structure formation [3], and the observed spectrum of the cosmic microwave background $[4,5,6]$, indicates that about $26 \%$ of the total mass-energy of the Universe is non-baryonic and non-relativistic cold component, which is not subject to electromagnetic interactions. Despite the long history of observations the particle content of DM remains unknown $[7,8,9]$. One possible solution would be the existence of new particles beyond the Standard Model.

Among many candidates for DM particles the most favored are the so-called WIMPs (weakly interacting massive particles) with a mass in the $\mathrm{GeV}$ to $\mathrm{TeV}$ range $[10,11]$. The WIMPs existences are well motivated, as provide solutions for outstanding issues in both cosmology and particle physics. These particles appear in extensions to the Standard Model such as supersymmetry with R-parity conservation, and freeze out in the early Universe with an abundance that matches cosmological observations. Now DM particles can be detected in the laboratory experiments via elastic scattering off nuclei, causing recoils of a few $\mathrm{keV}$ in energy. However, no experimental indication for a standard WIMP was found in high sensitivity direct 
search experiments like EDELWEISS [12], XENON100 [13], LUX [14], SuperCDMS [15] for more than a few tens of $\mathrm{GeV} / \mathrm{c}^{2}$ mass.

Thermal relics with masse below $100 \mathrm{GeV} / \mathrm{c}^{2}$ are also disfavored both from constraints set by Fermi-LAT searches for annihilation signals from Milky Way dwarf galaxies [16], and from the impact that WIMP annihilation would leave on the cosmic microwave background anisotropies [17]. Furthermore search for dark matter particles directly produced in protonproton collisions in the CERN LHC experiments ATLAS and CMS did not show any indications of such particles so far [18, 19, 20].

On the other hand in the region of light WIMPs below $30 \mathrm{GeV} / \mathrm{c}^{2}$, some experiments such as DAMA/LIBRA [21], CoGeNT [22], CRESST [23], CDMS II Si [24] claim or are interpreted as finding dark matter signals.

It is obvious that for observation of low-energy WIMP-induced nuclear recoils, detectors with both low detection threshold and a very low background are needed. The sensitivity of a dark matter direct detection experiment depends on the WIMP mass and on the nature and strength of its coupling to atomic nuclei.

Alternative to the quest of WIMP particles is a search of ultralight DM particles such as neutrino, dark photons or axions. Again, there is no indication that the high-energy neutrinos originating from dark matter annihilations in the Sun, which can escape and be observed by neutrino telescopes, such as Baksan [25], Super-Kamiokande [26], ANTARES [27] and IceCube [28]. When arguing the existence of Dark Matter (DM), which exceeds the mass of the visible Universe by about five times, with the help of light particles, the component can be light, weakly interacting, pseudoscalar bosons - axions $\left(\mathrm{m}_{\mathrm{a}} \sim 1 \div 1000 \mu \mathrm{eV}\right)[29]$.

Axions were introduced as a consequence of an extension of the Standard Model. The expansion, in particular, follows from the desire to preserve $\mathrm{CP}$ invariance, which is violated by the absence of an electric dipole moment in the proton, and axions, as particles of a new field that preserves CP invariance, play this role [30-32]. Moreover, new particles have no charge. Unexpectedly, it turned out that astrophysical and cosmological problems of the energy balance when calculating the emission budget of stars, the existence of halos around galaxies [33, 34], the cooling processes of white dwarfs [35], and the shortening of the duration of the SN 1987A supernova explosion [36, 37] can also be described more simply, using the existence of axions. (According to estimates $[29,37]$, the particle density is $\sim 10^{21} \mathrm{~m}^{-3}$ ). Thus, the axions that make up the DM can be detected not only at accelerators, but also in laboratories studying cosmic radiation, since it serves as a source of axions [38, 39].

The mechanism of the conversion of an axion into two photons in an external magnetic field (Primakov's mechanism), proposed earlier to explain the decay of muons, was chosen as a 
mechanism convenient for registering axions [40]. The reverse process of transformation of photons into axions is also possible. In addition, in the laboratory it is possible to use sources of powerful coherent radiation (lasers) and with their help simulate the conversion of photons into axions, as well as the reverse process. An additional impetus to research on the conditions for observing axions was the applied problem of detecting the passage of an axion beam through the thickness of matter without a significant weakening of its intensity - the LSTW (light shining through walls) experiment [41]. It was proposed to use a small interaction cross section to establish communication through massive opaque obstacles, for example, the Earth or Earth Moon.

As noted above, the Primakov's mechanism uses low-frequency virtual photons of the magnetic field that resonate with the field of axions. The probability of the axion-photon conversion is $\mathrm{p} \sim$ $\left(g B_{0} l\right)^{2}$ - here $g$ is the constant of the photon-axion coupling. Strong enough magnetic fields $B_{0}$ $\left(B_{0} \sim 10^{2} \mathrm{~T}\right)$ along the entire path $l\left(\sim 10^{2} \mathrm{~m}\right)$, where the conversion takes place as well as intense laser beams $\left(\sim 10^{21} \mathrm{~W} / \mathrm{cm}^{2}\right)$ for such reactions are required [42]. Because during solar flares on Sun surface there are a lot of photons piercing magnetic field, then it is possible to observe axion generation by helioscope (IAXO project) [43].

In recent time some investigators expect the time intervals measurements are keys for DM observation $[44,45]$.

\section{Non-corpuscular "ether wind" and possible registration of its pressure by the SQUID-magnetostrictor system}

Here we consider a new approach for DM description, based on the possibility that DM and Dark Energy (DE), can be considered as two different aspects of the same cosmological essence, named "Dark Substance" (DE - hypothetical pervasive substance, which can be responsible for the additional relative acceleration in the Hubble law of galaxies recession). The means of approach can be anticipated from integrated Sachs - Wolfe effect. It points on a correlation between anisotropy of local equilibrium temperature for CMB (i.e. fluctuations of photons) and large scale Universe structure, or in accordance with Einstein GR, of gravitational potential due to the massive particles that do not otherwise interact, the DM. And both photons and DM are in primordial soup - DE. So the temperature fluctuation is directly related to the gravitational potential fluctuation by some constant [46]. The long-scale structure depends on a matter density, the CMB anisotropy - defines by fluctuations of a cosmological gravitational potential, which changes the photon frequency. The effect is in a good agreement with the prediction of the concordance $\Lambda \mathrm{CDM}$ cosmology [47-49]. 
According to an offered model, with DE density of about $300 \mathrm{TeV} / \mathrm{m}^{3}$, it represents the unperturbed state of "Dark Substance", while its folds or perturbations play the role of elementary DM heavy particles. These particles will be stable if all their decay channels into any combination of other particles are blocked, or also, in our case, if their potential will have local minima, i.e., local traps providing metastable excited states. The Hamiltonian with metastable traps can be represented, for example, as follows:

$$
\mathrm{H}=\frac{1}{2}\left(\frac{\partial \varsigma}{\partial x}\right)^{2}+\frac{1}{2 c^{2}}\left(\frac{\partial \varsigma}{\partial t}\right)^{2}+\alpha \varsigma^{*} \varsigma-\beta \cos \gamma \sqrt{\varsigma^{*} \varsigma} .
$$

The nonlinear wave equation, corresponding to this Hamiltonian, and describing the dynamics of perturbations to Dark Substance will be like the "quasi-sine-Gordon" equation

$$
\frac{\partial^{2} \varsigma}{\partial x^{2}}-\frac{1}{c^{2}} \frac{\partial^{2} \varsigma}{\partial t^{2}}+\alpha \varsigma^{*}+\frac{1}{2} \beta \gamma \sqrt{\varsigma^{*} / \varsigma} \sin \gamma \sqrt{\varsigma^{*} \varsigma}=0
$$

Moreover, the nonlinear potential $\Pi(\varsigma)=\alpha \varsigma^{*} \varsigma-\beta \cos \gamma \sqrt{\varsigma^{*} \varsigma}$ appearing in (1), has the analytic structure very similar to the "parabolic washboard potential" [50] used to describe metastable states in the superconducting ring of a SQUID with one Josephson junction, namely:

$$
\Pi(\varsigma)=\alpha|\varsigma|^{2}-\beta \cos \gamma|\varsigma| \Leftrightarrow E(\Phi)=\frac{1}{2 L} \Phi^{2}-I_{C} \Phi_{o} \cos \frac{2 \pi}{\Phi_{o}} \Phi,
$$

where $\Phi$ is the magnetic flux piercing the superconducting ring and $L$ is the inductance of the circuit itself. We represent the profile of the washboard potential in figure 1. Some comments about this figure are needed. For small $\varsigma$ (when the disturbance has not yet reached the first trap) folds of the Dark Substance have a quasi-harmonic character, and their quanta will have a mass $m=\frac{\hbar}{c} \sqrt{2 \alpha+\beta \gamma^{2}}$. However, such particles may be unstable, and the rate of decay from classical positions will correspond to the viscosity of the Dark Substance [51, 52]. At large amplitudes of $\varsigma$, the disturbance at some moment will "catch" the lowest energy trap. The trajectory of the oscillation $\varsigma$ will now be a circle in the plane $\left\{\varsigma, \varsigma^{*}\right\}$, corresponding to a local minimum of potential. The rotation around the circumference of a local minimum is similar to the mechanism of occurrence of massless Goldstone bosons in Weinberg-Salam's model. However, in this example, the mass of excitations ("zero energy") is determined by the height of the bottom of the trap with respect to the main vacuum state $\varsigma 0 \varsigma^{*}=0$, and will be non-zero. The stability of such excitations, playing (in this example) the role of DM particles, is guaranteed by the height of the wall of the potential well, occurring in the vicinity of the local potential minimum. 


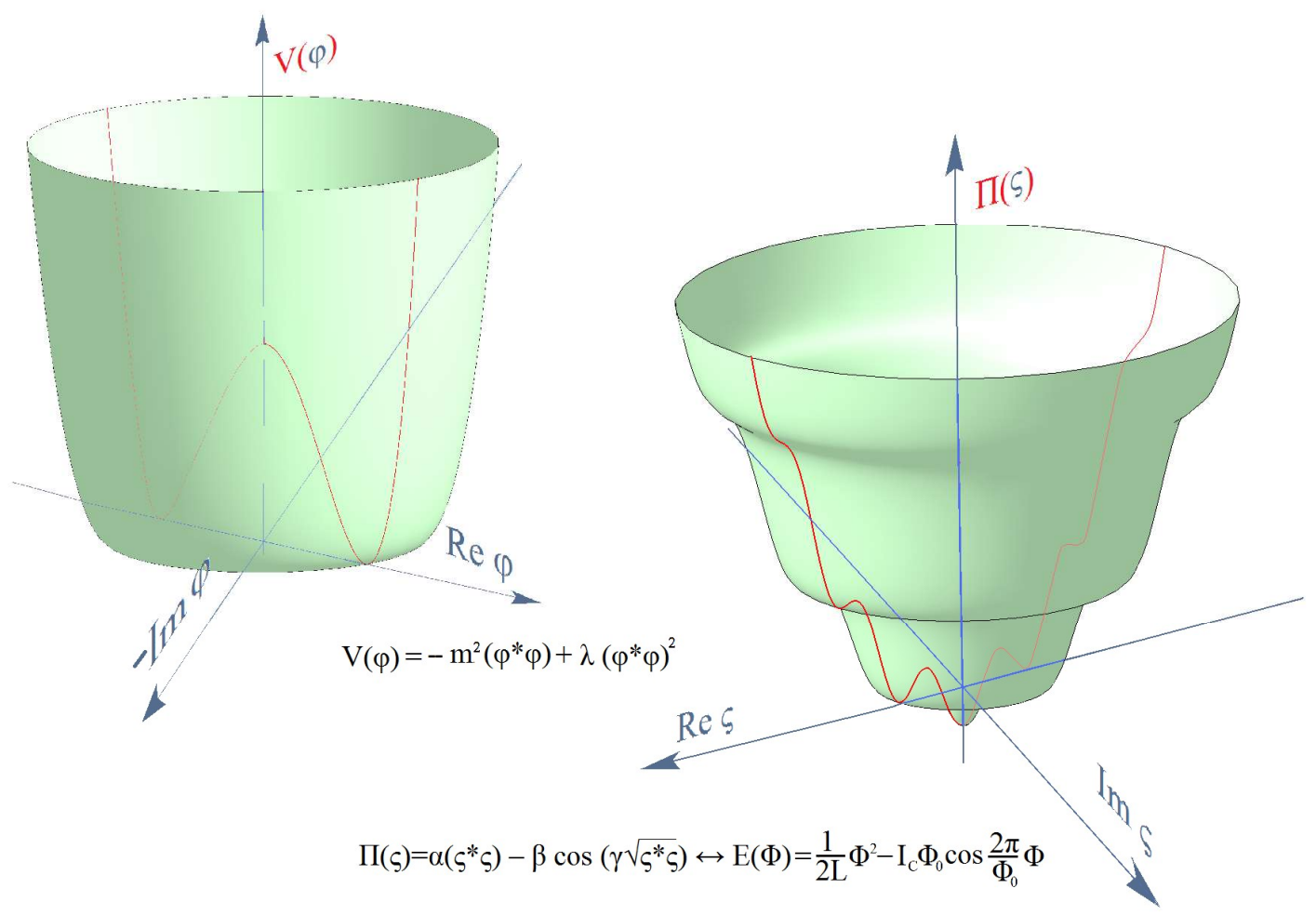

Fig 1. On the right, the washboard potential, characterized by local minimum positions, which are the metastable states of DM particles, is shown. The lower local minimum is associated with a light (or also hot) component of DM, and the top one with a heavy (or also cold) component of DM. The absolute minimum lies in the region of negative energies and is associated with antigravitational properties of DE. On the left (for comparison) the potential used in models of spontaneous symmetry breaking is displayed.

Therefore, the search of DM particles, as stable moving excitations of Dark Substance, may be intimately connected with the research of the action of the DE non-corpuscular flux on the ordinary matter. By knowing that the free mean path is connected to the cross section of interaction by the relation $\ell^{*} \approx 1 /\left(\sigma n_{A}\right)$, we may say that DE transfers to a slab of material, consisting of ordinary atoms of concentration $n_{A}$, with area $S$ and "maximum depth" $\ell$, a momentum $q=\ell^{*} S \rho_{D E} / v$, where $v$ is the DM particles speed relative to the substance. In this way, Dark Substance exerts the pressure

$$
p^{*}{ }_{D E}=F / S=\left(\ell^{*} S \rho_{D E} / v\right) /\left(\ell^{*} / v\right) / S=\rho_{D E}
$$

on the slab. Thus, the effective pressure drop across the length $\ell$ is estimated as $p_{\mathrm{DE}}=p^{*}{ }_{D E}$ $\ell / \ell^{*}=\rho_{D E} \ell \sigma n_{A}$. In accordance with the generally accepted value of the average density of Dark Energy $\rho_{D E} \approx 300 \mathrm{TeV} / \mathrm{m}^{3}$, taking into account the above-obtained "optimistic" estimation of the interaction cross section $\sigma \approx 10^{-35} \mathrm{~cm}^{2}$, the pressure drop across a one-meter barrier, having a concentration of atoms $n_{A} \approx 3 \times 10^{22} \mathrm{~cm}^{-3}$, will be of the order of $p_{D M}(\ell=1 \mathrm{M}) \approx 7 \times 10^{-16} \mathrm{~Pa}$. 
In order to register such pressure, a dynamometer that performance capable to fix the strength of $10^{-16} \mathrm{~N}$ at the end of the cylinder, which dimensions $\ell \times S \approx 1 \mathrm{~m} \times 0.15 \mathrm{~m}^{2}$, is required. Apparently, the suitable candidate for the role of the super-high-sensitivity dynamometer is the SQUIDmagnetostrictor system [53-55] which has been previously supposed to be used in projects for the detection of gravitational waves, etc. (Figure 2).

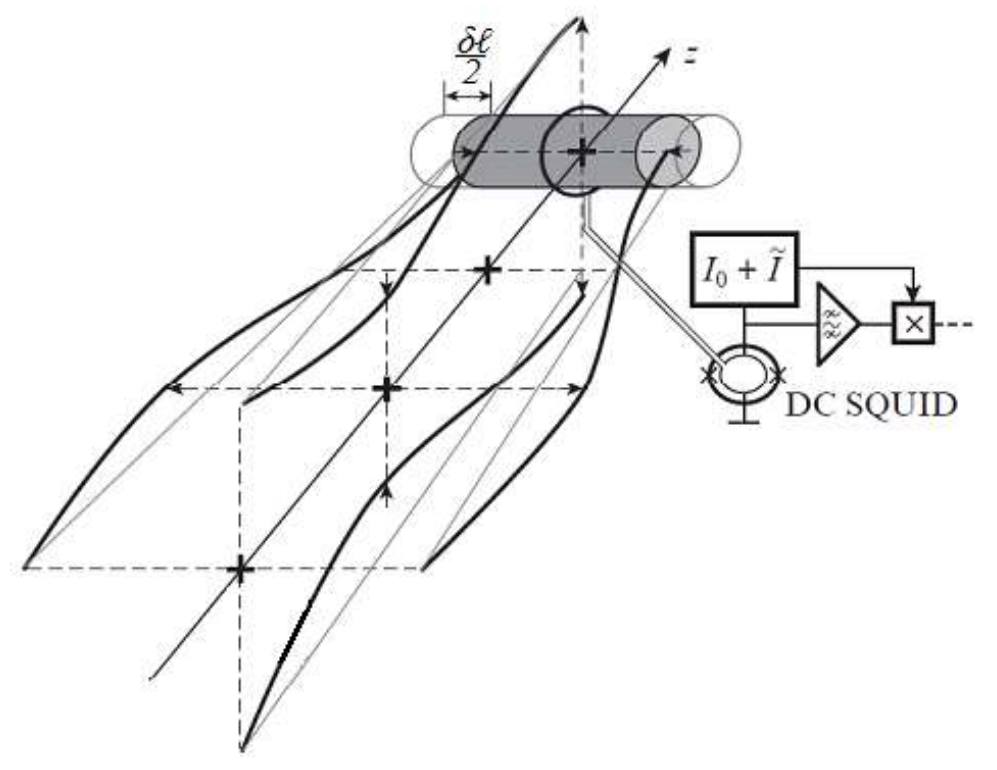

Fig 2. Schematic view of the SQUID-magnetostrictor system for the detection of gravitational waves (the magnetostrictive cylinder is represented in grey) [55].

Ultra-high sensitivity is achieved by means of this system. In fact, high strain-gauge effectiveness of the sensor can be achieved, since it operates on the principle of the reverse magnetostrictive effect, generated, in its turn, by collective quantum solid-state effects [61]. On the other hand, the high ("quantum scale" level) sensitivity of SQUID systems, used as measuring instruments, allows accurate registration of events.

The physical quantity describing the reverse magnetostrictive effect (discovered by Emilio Villari in 1865) in a particular material is the ratio of the internal magnetic induction field to the growth of its outside pressure, i.e., $\Lambda^{-1}=\frac{\partial B}{\partial P}$. For example, an alloy made up of $54 \% \mathrm{Pt}$ and $46 \%$ Fe, with $\mu \approx 14000$, will have $\Lambda^{(-1)} \approx 10^{-4} \mathrm{~T} / \mathrm{Pa}$ (which is basically not a record value). Thus the magnetic response, measured by the SQUID, is related to the force action $\delta \mathrm{F}$ by this expression $\delta \Phi=\Lambda^{(-1)} \delta \mathrm{F}$. Accordingly, the capability to register the pressure of non-corpuscular Dark Matter flow, estimated above for $\sigma \approx 10^{-35} \mathrm{~cm}^{2}$ at $\delta \mathrm{F} \approx 10^{-16} \mathrm{~N}$, requires an "absolute" (not reduced to the time of the signal accumulation) SQUID sensitivity for the magnetic flux of the order of 
magnitude of $10^{-20} \mathrm{~Wb} \approx 5 \times 10^{-6} \Phi_{0}$. The actual value of a good DC-SQUID is of about $\delta \Phi \approx 10^{-6}$ $\Phi_{0} / \sqrt{\mathrm{Hz}}$, which provides the desired sensitivity with a margin of approximately 2 orders of magnitude (at least) due to the possibility of a 3-hour signal accumulation.

\section{Conclusions}

In this paper, starting from an introduction about DM and its cosmological properties, we have focused our attention on a brief description of a toy model unifying DE and heavy DM component. In the context of "unifying" trend, clearly dominant in the modern elementary particle physics, we have also proposed a simple unimodel, where we try to consider the corpuscular Dark Matter (DM) and non-corpuscular Dark Energy (DE) from single approach. This is the proposed model, in which the DE is an absolutely continuous substance, playing the role of a room for metastable excitations, which can be identified as Dark Matter particles. From this point of view, we cannot assume DE as a medium suitable for the role of absolute spacetime, relative to which the states of other objects are considered. DE is rather an active medium, one of the manifestations of the activity of which is the effect of the production of DM particles.

The dual form of the DE entity gives possibilities for experimental research. Estimates, carried out within that model, indicate the possibility of detection of the "ether wind" pressure, created by the non-corpuscular incoming flow, corresponding to the galactic orbital motion of the Earth. It is argued that these types of investigations could be performed by using the SQUIDmagnetostrictor experimental system. At the same time, the concept of quantizing the continuity of the medium, which was used in the article, should undergo some modification in order to establish the boundaries of possible quantization both in the energy scale and in the spatial one.

\section{References}

[1] G. Bertone, D. Hooper, A history of dark matter, arXiv:1605.04909.

[2] V. C. Rubin, N. Thonnard, and W. K. Ford Jr., "Rotational properties of $21 \mathrm{SC}$ galaxies with a large range of luminosities and radii, from NGC 4605/R $=4 \mathrm{kpc}$ to UGC $2885 / \mathrm{R}=122 \mathrm{kpc}$ ", Astrophys. J. 238 (1980) 471, doi:10.1086/158003.

[3]V. Springel et al., "Simulating the joint evolution of quasars, galaxes and their large-scale distribution”, Nature 435 (2005) 629, doi:10.1038/nature03597, arXiv:astro-ph/0504097.

[4]WMAP Collaboration, "Wilkinson Microwave Anisotropy Probe (WMAP) three year results: implications for cosmology”, Astrophys. J. Suppl. 170 (2007) 377,doi:10.1086/513700, arXiv:astro-ph/0603449. 
[5] Planck Collaboration, "Planck 2013 results. XVI. Cosmological parameters", Astron. Astrophys. 571 (2014) A16, doi:10.1051/0004-6361/201321591, arXiv:1303.5076.

[6] Planck Collaboration, P. A. R. Ade et. al., Planck 2015 results. XIII. Cosmological parameters, 1502.01589 .

[7] L. Bergstrom, Nonbaryonic dark matter: Observational evidence and detection methods, Rept.Prog.Phys. 63 (2000) 793.

[8] G. Bertone, D. Hooper and J. Silk, Particle dark matter: evidence, candidates and constraints, Phys. Rep. 405, 279 (2005).

[9] K.A. Olive et al. (Particle Data Group), Chinese Phys.C38, 090001 (2014).

[10] Ryabov V.A., Tsarev V.A., Tskhovrebov A.M. , The search for dark matter particles, Physics- Uspekhi 51 (11), 1091 (2008)

[11] J.L. Feng, Dark matter candidates from particle physics and methods of detection, Ann. Rev. Astron. Astrophys. 48 (2010) 495.

[12] Armengaud E., et al, (EDELWEISS Collaboration), Performance of the EDELWEISS-III experiment for direct dark matter searches. JINST 12 (2017) no.08, P08010 [arxiv:1706.01070] doi: 10.1088/1748-0221/12/08/P08010.

[13] E. Aprile et al. (XENON100 collaboration), Phys. Rev.Lett. 109, 181301 (2012).

[14] D.S. Akerib et al. (LUX collaboration), Results of a Search for Sub-GeV Dark Matter Using 2013 LUX Data,” Phys. Rev. Lett. 122, 13101 (2019), arXiv:1811.11241

doi::https://doi.org/10.1103/PhysRevLett.122.131301.

[15] R. Agnese et al. (SuperCDMS collaboration), Search for Low-Mass Weakly Interacting Massive Particles with SuperCDMS, Phys. Rev.Lett. 112, 241302 (2014).

doi: https://doi.org/10.1103/PhysRevLett.112.241302.

[16] Fermi-LAT Collaboration, M.N. Mazziotta et al. Search for dark matter signatures in the cosmic-ray electron and positron spectrum measured by the Fermi Large Area Telescope .PoS ICRC2019 (2020) 531 doi:10.22323/1.358.0531

[17]Planck Collaboration, P.A.R. Ade et al., Planck 2015 results. XIII. Cosmological parameters [arxiv:1502.01589].

[18] G. Aad et al. (ATLAS), Search for new phenomena in final states with an energetic jet and large missing transverse momentum in pp collisions at $\mathrm{s} V=8 \mathrm{TeV}$ with the ATLAS detector, Eur. Phys. J. C75, 299 (2015).

[19] (ATLAS Collaboration). Search for resonances in the mass distribution of jet pairs with one or two jets identified as b-jets in proton--proton collisions at $\mathrm{s} V=13 \mathrm{TeV}$ with the ATLAS detector. e-print arXiv:1805.09299 doi: https://arxiv.org/abs/1805.09299 (2018). 
[20]V. Khachatryan et al. (CMS Collaboration), Search for new physics in top quark production with additional leptons in proton-proton collisions at $s \downarrow=13 \mathrm{TeV}$ using effective field theory arXiv:2012.04120; CMS-TOP-19-001; CERN-EP-2020-211; CMS-TOP-19-001-003.- 2020. - 50 p.

[21] P. Belli, R. Bernabei, F.A. Danevich et al. (DAMA Collaboration. Eur. Phys. J. A 55 (2019) 140 [arXiv:1908.11458] doi: 10.1140/epja/i2019-12823-2 .

[22] C. E. Aalseth et al. (CoGeNT collaboration), Results from a Search for Light-Mass Dark Matter with a p-Type Point Contact Germanium Detector, Phys.Rev.Lett. 106, 131301 (2011). doi: https://doi.org/10.1103/PhysRevLett.106.131301

[23], G. Angloher, M. Bauer, I. Bavykina, et al. (CRESST Collaboration): Dark-Photon Search using Data from CRESST-II Phase 2. (2017). Eur Phys J C 77:299.

doi: 10.1140/epjc/s10052-017-4878-6, arXiv:1612.07662 .

[24] R. Agnese et al. (superCDMS Collaboration) Search for Low-Mass Dark Matter with CDMSlite Using a Profile Likelihood Fit. Phys. Rev. D 99, 062001 (2019) doi:10.1103/PhysRevD.99.062001.

[25] M. M. Boliev et al., Search for muon signal from dark matter annihilations in the Sun with the Baksan Underground Scintillator Telescope for 24.12 years, JCAP 1309 (2013) 019.

[26] Y. Suzuki. The Super-Kamiokande experiment/ Eur. Phys. J. C. 79:298 (2019) doi: https://doi.org/10.1140/epjc/s10052-019-6796-2.

[27] A.Albert,M.André, M.Anghinolfi, et al. Search for dark matter towards the Galactic Centre with 11 years of ANTARES data. Phys. Lett. B 805135439 (2020).

doi: https://doi.org/10.1016/j.physletb.2020.135439.

[28] R. Abbasi, M. Ackermann, J. Adams et al. Search for GeV Neutrino Emission During Intense Gamma-Ray Solar Flares with the IceCube Neutrino Observatory. arXiv:2101.00610v1 Submitted to Physical Review D.

[29] J.V. Sloan, M. Hotz, D.B.Tanner, K. van Bibber et. al. Limits on axion-photon coupling or on local axion density: Dependence on models of the Milky Way's dark halo. Physics of the Dark Universe 14, pp. 95-102 (2016). doi: 10.1016/j.dark.2016.09.003.

[30] R. D. Peccei and H. R. Quinn "CP Conservation in the Presence of Pseudoparticles". Phys. Rev. Lett. 38, 1440-43 (1977) doi: 10.1103/PhysRevLett.38.1440.

[31] S.Weinberg "A New Light Boson? ", Phys. Rev. Lett. 40, 223-6 (1978).

doi: 10.1103/PhysRevLett.40.223.

[32] F. Wilczek. Problem of strong P and T invariance in the present of instantons. Phys. Rev. Lett. 40, 279-82 (1978) doi:10.1103/PhysRevLett.40.279.

[33] P. Sikivie. Experimental Tests of the "Invisible" Axion. Phys. Rev. Lett. 51, 1415 - (1983) doi: http://dx.doi.org/10.1103/PhysRevLett.51.1415 Erratum Phys. Rev. Lett. 52, 695 (1984). 
[34] G.G. Ruffelt. Astrophysical methods to constrain axions and other novel particle phenomena. Phys.Rept. 198 pp.1-113 (1990) doi: 10.1016/0370-1573(90)90054-6.

[35] B. Melendez, M.M. Bertolami, and L. Althaus. Revisiting the Impact of Axions in the Cooling of White Dwarfs. arXiv:1210.0263v1 [hep-ph] 1 Oct 2012.

[36] Л.В. Окунь. Пределы на электродинамику: парафотоны? . ЖЭТФ 83, $892-5$ (1982); L.V. Okun. Limits on electrodynamics: paraphotons? Sov. Phys. JETP 56, 502-5 (1982).

[37] A. Ringwald. The hunt for axions. arXiv:1506.04259v1 [hep-ph] (2015).

[38] N. Du et al. (ADMX Collaboration). Search for Invisible Axion Dark Matter with the Axion Dark Matter Experiment. Phys. Rev. Lett. 120, 151301 (2018) doi:https://doi.org/10.1103/PhysRevLett.120.151301.

[39] E. Aprile et al. (XENON Collaboration) XENON1T Light Dark Matter Search with Ionization Signals in Phys. Rev. Lett. 123, 251801 (2019) doi:https://doi.org/10.1103/PhysRevLett.123.251801.

[40] H. Primakoff. Photo-production of neutral mesons in nuclear electric fields and the mean life of the neutral meson. Phys. Rev. 81, 899 (1951).

[41] P. Sikivie, D. Tanner, K.van Bibber "Resonantly Enhanced Axion-Photon Regeneration". Phys. Rev. Lett. 98 (17): 172002 (2007) arXiv:hep-ph/0701198. doi:10.1103/PhysRevLett.98.172002

[42] В.С. Горелик, Г.Н. Измайлов "Стимулированная конверсия фотонов в псевдоскалярные бозоны" Краткие сообщения по физике 38, №6, стр. 39-49 (2011).

[43] I. G. Irastorza, M. Pivovaroff, and H. T. Kate. IAXO: the International Axion Observatory. CERN Courier (August 2014) https://cerncourier.com/a/iaxo-the-international-axionobservatory.

[44] C. J. Kennedy, E. Oelker, J. M. Robinson et al. Precision Metrology Meets Cosmology: Improved Constraints on Ultralight Dark Matter from Atom-Cavity Frequency Comparisons. Phys. Rev. Lett. 125, 201302 (2020) doi: https://doi.org/10.1103/PhysRevLett.125.201302.

[45] E. Pedrozo-Peñafiel, S. Colombo, Chi Shu, V. Vuletić et al. Entanglement on an optical atomic-clock transition. Nature 588, p.414-418 (2020).

doi: https://doi.org/10.1038/s41586-020-3006-1.

[46] R. K. Sachs, A. M. Wolfe. Perturbations of a Cosmological Model and Angular Variations of the Microwave Background. Astrophys. J., 147, p.73 (1967). doi:10.1086/148982 .

[47] A. Maeder. An alternative to the $\Lambda \mathrm{CDM}$ model: the case of scale invariance. The Astr. J, 834, 2, p. 194 (2017).

[48] A. J. Nishizawa. Integrated Sachs Wolfe Effect and Rees Sciama Effect. Progress of Theoretical and Experimental Physics, 2014, 6, 06B110 (2014) doi: 10.1093/ptep/ptu062

[49] A. Kovács. The part and the whole: voids, supervoids, and their ISW imprint. Monthly Notices of the Royal Astronomical Society, 475, 2, (2018), p. 1777-1790. https://doi.org/10.1093/mnras/stx3213. 
[50] A. Barone, G. Paternò. Physics and applications of the Josephson effect. Wiley (1982), p. 529.

[51] H. Velten, D J. Schwarz, J.C. Fabris, W Zimdahl. Viscous dark matter growth in neo-

Newtonian cosmology. Phys. Rev. D 88, 103522, doi: 10.1103/PhysRevD.88.103522. (2013).

[52] H. Velten, J. Wang, X. Meng. Phantom dark energy as an effect of bulk viscosity. Phys.

Rev. D 88, 123504, doi: 10.1088/0004-638X/ (2013).

[53] A.I.Golovashkin, A.V.Gudenko, L.N.Zherikhina, O.M.Ivanenko, K.Yu.Mitsen, A.M.Tskhovrebov. Fluctuation limit of measurements of the relative elongation of a magnetostrictive cylinder. JETP letters 60(8), 612 (1994).

[54] G.N. Izmailov, L.N.Zherikhina, V.A. Ryabov, A.M. Tskhovrebov. Dark Energy: The Nature and Feasibility of Laboratory Registration using SQUID-Magnetostrictor System in "Dark Energy: Theories, Developments, and Implications" Eds: K.Lefebvre, R.Garcia. Nova Sc. Pub.. doi: 10.3103/S1068335612090048, (2010).

[55] A.I.Golovashkin, L.N.Zherikhina, A.M.Tskhovrebov, G.N.Izmailov "Supersensitive SQUID/magnetostrictor detecting system", Quantum Electronics 42(12), 1140-1146 (2012), DOI: 10.1070/QE2012v042n12ABEH000000 (13 June 2012). 